\title{
Effect of theophylline on exercise capacity in COPD patients treated with combination long-acting bronchodilator therapy: a pilot study
}

This article was published in the following Dove Press journal:

International Journal of COPD

30 March 2012

Number of times this article has been viewed

\author{
Nha Voduc' \\ Gonzalo G Alvarez' \\ Kayvan Amjadi' \\ Caroline Tessier ${ }^{2}$ \\ Elham Sabri ${ }^{3}$ \\ Shawn D Aaron' \\ 'University of Ottawa, Division of \\ Respirology, ${ }^{2}$ The Ottawa Hospital \\ (General Campus), ${ }^{3} \mathrm{OHRI}$ Methods \\ Centre, Ottawa, ON, Canada
}

Correspondence: Nha Voduc

The Ottawa Hospital (General Campus), 50I Smyth Road, Box 21I, Ottawa, ON, Canada KIH 8L6

$\mathrm{Tel}+\mathrm{I} 6137378198$

Fax + I 6137378537

Email nvoduc@toh.on.ca
Background: Many patients with chronic obstructive pulmonary disease continue to experience significant functional limitation despite the use of both long-acting anticholinergic and betaagonist inhalers. Theophylline is a widely available medication which may further improve lung function and exercise performance. Previous studies evaluating the effects of theophylline on exercise capacity in chronic obstructive pulmonary disease (COPD) have demonstrated heterogeneous results.

Methods: We performed a randomized placebo-controlled double-blind pilot study assessing the effects of theophylline on constant load exercise duration and lung function, involving 24 COPD patients already treated with long-acting inhaled beta-agonist and long-acting anticholinergic bronchodilator therapy.

Results: Analyzable data was available in 10 of 12 subjects in the treatment arm and 11 of 12 subjects in the control arm. Theophylline was associated with a $26.1 \%$ (95\% confidence interval [CI]: -17.3-69.5) improvement in exercise duration compared to placebo. Four of 10 treated patients demonstrated an improvement in exercise duration exceeding the minimum clinically important difference of $33 \%$, compared to 1 of 11 controls $(P=0.15)$. Furthermore, peak ventilation was reduced by $11.1 \%,(95 \%$ CI: $0.77-21.5)$ which may suggest improvements in gas exchange. There were no significant observed differences in resting lung function nor measures of dyspnea between the two treatment groups.

Conclusions: Our study demonstrated a trend, but not a statistically significant improvement in exercise duration and a reduction in peak ventilation with theophylline. Based on the observed mean differences and standard deviations in this pilot study, a randomized controlled trial would require 45 subjects in each arm to detect a significant change in exercise duration.

Keywords: theophylline, chronic obstructive pulmonary disease, exercise capacity

\section{Introduction}

Over the last decade, long-acting inhaled bronchodilators have become a mainstay of treatment for chronic obstructed pulmonary disease (COPD). Compared to their short-acting counterparts, long-acting bronchodilators have been shown to provide greater dyspnea relief, improved lung function and reduced exacerbation rates. ${ }^{1-3}$ Unfortunately, patients with severe COPD may continue to experience significant symptoms, despite use of both long-acting beta-agonists and anticholinergics. For these patients, it has been suggested that theophylline may be added ${ }^{4,5}$ although the evidence supporting the use of theophylline in a combination regimen is limited. Theophylline causes bronchodilation by increasing cyclic adenosine monophosphate via the inhibition of phosphodiesterase. It has been shown that theophylline may 
reduce symptoms and hyperinflation, and improve exercise duration in patients with COPD. ${ }^{6}$ Furthermore, theophylline is inexpensive and easy to administer. Despite its advantages however, the use of theophylline is limited by its potential toxicities ${ }^{7}$ and drug interactions.

Few studies have evaluated the effects of theophylline in combination with long-acting bronchodilators. Zuwallack et $\mathrm{al}^{8}$ evaluated the effect of theophylline in combination with salmeterol. In those patients able to tolerate theophylline, theophylline with salmeterol produced greater improvements in forced expiratory volume in 1 second $\left(\mathrm{FEV}_{1}\right)$ and dyspnea than either therapy alone. Cazzola and Matera ${ }^{9}$ published the only study evaluating the effects of theophylline, in combination with both long-acting anticholinergics and beta-agonists (tiotropium and formoterol). They found no significant improvement in $\mathrm{FEV}_{1}$ with the use of theophylline but acknowledged that their study may have been underpowered.

It is possible that $\mathrm{FEV}_{1}$ may not be a sufficiently sensitive measure of bronchodilator response. A previous study by McKay et al ${ }^{10}$ using constant load treadmill testing demonstrated that theophylline improved exercise duration by $48 \%$ in patients with severe COPD. Tsukino et al ${ }^{11}$ demonstrated statistically significant but relatively smaller effects of theophylline on exercise capacity using an incremental cycle ergometer protocol. The Tsukino study did suggest that the effects of theophylline were additive to ipratropium. The McKay and Tsukino studies were able to demonstrate significant results despite enrolling only 20 and 21 patients, respectively, although other studies did not find any effect of theophylline on exercise capacity. ${ }^{12}$

The heterogeneity of previous study results makes sample size predictions for a randomized, controlled trial on this topic challenging. Consequently we designed a pilot study evaluating the effects of theophylline on constant load exercise duration in COPD patients already treated with both long-acting anticholinergic and beta-agonist inhalers. The goals of the study included an assessment of not only the effect of theophylline on constant exercise duration but the range (standard deviation) of response in this patient population. It is hoped that these results would inform the design of future studies.

\section{Methods}

We performed a prospective randomized double-blinded placebo controlled trial evaluating the short-term (4 weeks) effects of theophylline on exercise duration and lung function in patients already receiving both tiotropium and a long-acting beta-agonist (salmeterol or formoterol). Twenty-four patients with moderate-to-severe COPD who were already using chronic tiotropium and long-acting beta agonists were randomized to receive either: (a) 4 weeks of oral theophylline dosed to achieve therapeutic blood levels, or (b) identicalappearing placebo capsules.

Patients were recruited from the outpatient practices of academic respirologists in a tertiary care setting. All patients had to have a clinical diagnosis of COPD from their referring physician as well as an $\mathrm{FEV}_{1}$ of $<60 \%$ predicted and $\mathrm{FEV}_{1} /$ forced vital capacity [FVC] ratio $<70 \%$, and no significant reversibility with short-acting bronchodilator $(<12 \%$ change in $\mathrm{FEV}_{1}$ ). All patients had to be on stable doses of tiotropium $18 \mu \mathrm{g}$ once daily (qd) and either salmeterol $50 \mu \mathrm{g}$ twice daily (bid) or formoterol $12 \mu \mathrm{g}$ bid for at least 2 months. The use of inhaled corticosteroids was permitted as long as there were no dose changes over the last 2 months. All patients had to be clinically stable, with no exacerbations within the previous 2 months.

Exclusion criteria included the presence of lung disease other than COPD, any comorbidity that could potentially affect exercise capacity or ability to tolerate theophylline, and any medication that could interact with theophylline. Patients who were currently using theophylline or had used theophylline in the past were excluded as this could potentially bias the results. Patients on long-term oral steroids or who were concurrently participating in a pulmonary rehabilitation program were also excluded as these factors could potentially affect peripheral muscle function. Patients unable to demonstrate evidence of either respiratory or cardiac limitation on cardiopulmonary exercise testing as defined in the American Thoracic Society/American College of Chest Physicians statement on cardiopulmonary exercise testing ${ }^{13}$ were also excluded.

Study participation consisted of three visits for pulmonary function testing and exercise testing, with 1-3 additional short visits in-between for monitoring bloodwork.

\section{Visit I}

After confirmation of eligibility and informed consent, patients underwent full pulmonary function testing followed by an incremental cardiopulmonary exercise test to assess maximal work rate. Incremental exercise testing was performed on an electronically braked cycle-ergometer, using 20 watt increments every 2 minutes. The maximal work rate attained in the incremental test was used to calculate the load for subsequent constant load tests $(75 \%$ of the maximal workload). After a 30-minute recovery period, a practice 
constant load exercise test was performed to familiarize patients with the exercise protocol and minimize any learning effects for future tests.

\section{Visit 2}

Subjects returned within 1 week of visit 1. During this visit, the Baseline Dyspnea Index was administered and patients underwent repeat pulmonary function testing and the first constant load ( $75 \%$ of peak workload attained on incremental testing) exercise test. After completion of the constant load exercise test, subjects were then randomized to treatment with either placebo or theophylline therapy. Randomization was conducted using a computer generated variable-block algorithm. The patients and investigators (NV and CT) responsible for testing, data gathering, and data analysis were blinded to the theophylline allocation during the conduct of the study.

The form of theophylline used for this study was a 24-hour sustained release formulation. Theophylline was initially administered at doses of 400,600, or $800 \mathrm{mg} \mathrm{qd}$, depending on body weight $(10 \mathrm{mg} / \mathrm{kg}$ of body weight, with a $25 \%$ dose reduction for patients aged $>65$ years). Evening dosing was recommended so that peak blood levels would be present during the following morning. Both placebo and theophylline were administered in the form of identical capsules. The theophylline capsules used for the study were produced by overencapsulating half-tablets of sustainedrelease theophylline (200 per study capsule).

Regardless of randomization assignment, all subjects were asked to return for routine blood work within 5-7 days of starting the medication. Blood was drawn in the morning, at the time peak levels are expected (8-12 hours following the last dose of theophylline). Patients enrolled in the treatment arm had adjustments in their theophylline dose, depending on their blood level to achieve a blood level within the therapeutic range of 55-110 $\mu \mathrm{mol} / \mathrm{L}$. To maintain blinding, patients on placebo also underwent bloodwork and random sham-dosing adjustments were made to their placebo regimen. If dosing adjustments were required, repeat theophylline levels were measured 5-7 days after the dose change. This was repeated until bloodwork results were within the therapeutic range. Two physicians (GA and KA) were responsible for dosing adjustments and bloodwork monitoring. These investigators were unblinded and therefore were not involved in study data acquisition nor assessment of study outcomes.

\section{Visit 3}

Subjects returned for visit 3, 4 weeks after visit 2. On this visit, subjects had bloodwork to confirm that theophylline levels were within the therapeutic range. As with the second visit, subjects underwent pulmonary function testing as well as a constant load cardiopulmonary exercise test. Changes in symptoms were assessed using the transitional dyspnea index.

The study protocol was approved by the Ottawa Hospital Research Ethics Board (\#2006036-01H).

\section{Outcome measures}

The primary outcome measure was the percentage of change in the duration of constant load exercise (done at $75 \%$ of maximal work rate), measured in seconds from baseline (visit 2) compared to visit 3 for each treatment group. Secondary outcome measures included changes from visit 2 to visit 3 in expiratory flows $\left(\mathrm{FEV}_{1}, \mathrm{FVC}\right)$, dyspnea, and exercise responses (see Table 1).

\section{Sample size and statistical analysis}

As mentioned earlier, it was not possible to derive a reliable sample size from previous studies due to their heterogeneous results, hence the "pilot" nature of the study. Baseline data was summarized as means (standard deviation) by patient group. The primary outcome $(\%$ change in exercise duration between visits 2 and 3 ) in the two groups was compared using an unpaired Student's $t$-test. Secondary outcomes were compared with the Student's $t$-test or Wilcoxon signed-rank test where appropriate. Univariate correlational analyses were performed to assess for any relationship between baseline pulmonary function measures or exercise results and response to theophylline.

\section{Results}

Between May 2006 and March 2010, 27 patients underwent a visit 1 assessment. Three of 27 patients did not meet inclusion criteria and were not randomized (Figure 1).

Although the use of inhaled corticosteroids was not required, 23 of 24 patients were using inhaled corticosteroids in addition to tiotropium and a long-acting beta-agonist (Table 2). All enrolled subjects had at least Global Initiative for Chronic Obstructive Pulmonary Disease (GOLD) stage 3 COPD by spirometric criteria, with a mean $\mathrm{FEV}_{1}$ of approximately 30\% (range 15\%-47\%) (Table 2).

Three of the 24 randomized patients were excluded after randomization, two of whom were randomized to the theophylline arm. One theophylline patient developed intolerable nausea despite blood levels that were near the lower limit of the therapeutic range. The other theophylline patient was admitted to hospital with acute coronary syndrome, within 1 week 
Table I Pulmonary function and cardiopulmonary exercise results after treatment

\begin{tabular}{|c|c|c|c|c|}
\hline & Placebo $(n=I I)$ & Theophylline $(n=10)$ & Difference in means & $95 \% \mathrm{Cl}$ for Dif. \\
\hline Change in $\mathrm{FEV}_{1}, \%$ predicted & $1.82(3.52)$ & $0.70(3.20)$ & -1.12 & $(-4.20-1.96)$ \\
\hline Change in TLC, \% predicted & $-0.62(5.01)$ & $2.70(8.68)$ & 3.32 & $(-4.02-10.67)$ \\
\hline Change in RV, \% predicted & $-5.62(15.36)$ & $11.10(18.00)$ & 16.72 & $(-0.26-33.7 I)$ \\
\hline Change in DLCO, \% predicted & $-1.09(3.36)$ & $-1.30(7.21)$ & -0.21 & $(-5.62-5.20)$ \\
\hline Transitional dyspnea index & $0.27(2.33)$ & $1.20(2.20)$ & 0.93 & $(-1.15-3.00)$ \\
\hline \multicolumn{5}{|l|}{ Constant load exercise data } \\
\hline Change in exercise duration (seconds) & $20.36(67.62)$ & $62.70(163.00)$ & 42.34 & $(-78.69-163.40)$ \\
\hline Change in exercise duration ( $\%$ change) & $2.80(27.58)$ & $28.91(57.65)$ & 26.11 & $(-17.29-69.53)$ \\
\hline Change in $\mathrm{VO}_{2}, \%$ predicted & 4.09 (II.72) & $-1.60(10.25)$ & -5.69 & $(-|5.79-4.4|)$ \\
\hline Change in VE, \% predicted & $-0.36(13.15)$ & $-11.50(8.87)$ & -11.14 & $(-21.50-0.77)$ \\
\hline Change in HR, \% predicted & $1.45(4.70)$ & $3.80(5.09)$ & 2.35 & $(-2.13-6.82)$ \\
\hline Change in resting IC & $0.13(0.25)$ & $0.07(0.32)$ & -0.06 & $(-0.32-0.20)$ \\
\hline Change in peak IC & $0.03(0.30)$ & $0.14(0.31)$ & 0.11 & $(-0.17-0.39)$ \\
\hline \multicolumn{5}{|l|}{ Isotime exercise data } \\
\hline Change in $\mathrm{VO}_{2}(\mathrm{~mL} / \mathrm{min})$ & $0.08(0.22)$ & $-0.02(0.13)$ & -0.10 & $(-0.28-0.07)$ \\
\hline Change in HR (beats/min) & $-0.33(6.95)$ & $4.70(10.02)$ & 5.03 & $(-3.4 \mid-13.48)$ \\
\hline Change in VE (L/min) & $1.56(5.95)$ & $0.44(4.27)$ & -1.12 & $(-6.09-3.86)$ \\
\hline Change in IC (L) & $0.09(0.26)$ & $0.16(0.36)$ & 0.07 & $(-0.23-0.38)$ \\
\hline Change in dyspnea (Borg scale) & $-0.17(1.75)$ & $0.45(2.43)$ & 0.62 & $(-1.46-2.69)$ \\
\hline Change in leg fatigue (Borg scale) & $-0.33(1.66)$ & $0.90(2.42)$ & 1.23 & $(-0.80-3.27)$ \\
\hline
\end{tabular}

Note: Values in placebo and theophylline columns represent means, followed by standard deviations.

Abbreviations: $\mathrm{Cl}$, confidence intervals; DLCO, diffusing capacity; $\mathrm{FEV}_{1}$, forced expiratory volume in I second; FVC, forced vital capacity; HR, heart rate; IC, inhibitory capacity; RV, residual volume; TLC, total lung capacity; VE, ventilatory efficiency; $\mathrm{VO}_{2}$, oxygen consumption.

of randomization. Although the patient reported compliance with the study medication, blood levels drawn at the time of admission did not detect theophylline. One patient randomized to the placebo arm was excluded due to inability to provide maximal results on visit 2 exercise testing. As per protocol, this patient should have been excluded before randomization but this did not occur due to investigator error. The decision to exclude this patient from analysis was made before unblinding and data analysis. All other patients demonstrated evidence of ventilatory limitation during exercise testing.

All patients randomized to theophylline demonstrated blood levels within the target range at the time of visit 3 . The ten patients with complete data in the theophylline arm demonstrated a mean improvement of $28.9 \%$ (95\% confidence intervals $[\mathrm{CI}]:-12.3-70.2)$ in exercise duration, compared to $2.8 \%$ (95\% CI:-15.7-21.3) for the 11 patients in the placebo arm (Table 1). The between-group difference in change in exercise duration was $26.1 \%$ (95\% CI: - 17.3-69.5) or 42.3 seconds (95\% CI: $-78.7-163.4$ ). Given the wide standard deviation in exercise duration for the theophylline arm, the difference in exercise duration was not statistically significant $(P=0.22)$.

In addition to exercise duration, we also measured the effects of theophylline on other exercise parameters, symptoms, and lung function (Table 1). Exercise parameters, such as ventilation, inspiratory capacity and heart rate were compared at both peak exercise as well as isotime. Isotime analysis was conducted by comparing exercise parameters during the same time point. Isotime was determined by the shorter of the two exercise tests during visits 2 and 3. Theophylline was not associated with any improvements in resting lung function, however it was associated with a small but statistically significant reduction in peak ventilation (-11.1\%; 95\% CI: -0.77-21.5).

As noted above, there were two significant adverse events (intolerable nausea and acute coronary syndrome) in the theophylline group compared to none in the placebo group, although it is difficult to attribute the acute coronary syndrome to the use of theophylline, given undetectable blood levels at the time of presentation. Overall, side-effects were reported by five of 12 patients in the theophylline group, compared to two of 12 patients in the placebo group. Reported symptoms were abdominal cramping, headache, and insomnia. Three of 12 patients randomized to theophylline complained of abdominal cramping compared to none in the placebo group. Two patients on theophylline also complained of headaches compared to none in the placebo group. Two patients in each group complained of insomnia. Apart from the one patient who discontinued theophylline due to nausea, all other sideeffects were mild and self-limited. 


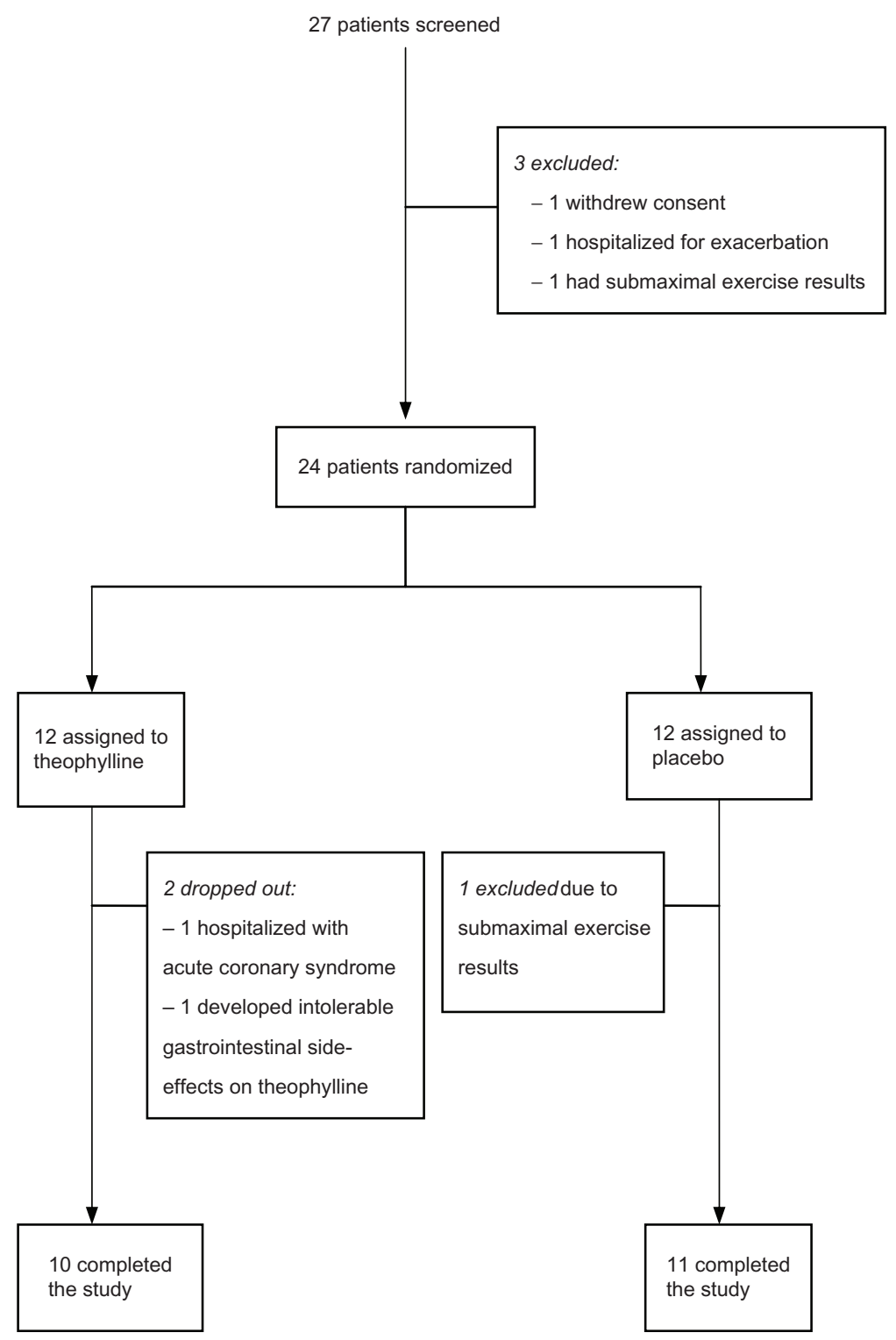

Figure I Patient flow diagram.

\section{Discussion}

Our study is the first to evaluate the effects of theophylline on exercise capacity in COPD patients already treated with combination long-acting inhaled bronchodilator therapy. We demonstrated that it is feasible to conduct a study evaluating theophylline in patients with severe COPD. The majority of the patients in our study were able to tolerate theophylline in doses sufficient to achieve what would be traditionally considered a therapeutic level. The dropout rate was only $12.5 \%$, which is comparable or lower than previous reports. However, the standard deviation in exercise duration for patients assigned to the theophylline arm is larger than that seen in patients assigned to placebo, and larger than seen with inhaled long-acting bronchodilator studies employing similar methodology. ${ }^{14-16}$

A recent study has suggested that the minimum clinically important difference (MCID) for constant load exercise is $33 \% .{ }^{17}$ We also analyzed the effects of theophylline on exercise duration by determining the proportion of subjects in each arm who demonstrated a MCID in constant load exercise duration. Four of 10 theophylline-treated patients demonstrated an improvement in exercise duration that was greater than the MCID, compared to only one of 11 placebo-treated patients. However, these results were also nonsignificant $(P=0.15)$.

Given the marked heterogeneity of the theophylline effects on exercise duration, we performed univariate analysis 
Table 2 Demographic and baseline data

\begin{tabular}{|c|c|c|c|c|}
\hline & Placebo $(n=I I)$ & $95 \% \mathrm{Cl}$ & Theophylline $(n=10)$ & $95 \% \mathrm{Cl}$ \\
\hline Age & 65 & & 65.5 & \\
\hline \multicolumn{5}{|l|}{ Sex } \\
\hline Male & $9(82 \%)$ & & $8(80 \%)$ & \\
\hline Female & $2(18 \%)$ & & $2(20 \%)$ & \\
\hline \multicolumn{5}{|l|}{ Medications } \\
\hline Tiotropium & II (I00\%) & & $10(100 \%)$ & \\
\hline Salmeterol & $8(73 \%)$ & & 7 (70\%) & \\
\hline Formoterol & $3(27 \%)$ & & $3(30 \%)$ & \\
\hline Inhaled corticosteroids & II (I00\%) & & $9(90 \%)$ & \\
\hline \multicolumn{5}{|l|}{ Pulmonary function } \\
\hline $\mathrm{FEV}_{1}, \%$ predicted & $29.54(11.25)$ & $(21.98,37.11)$ & $30.90(9.39)$ & $(24.18,37.61)$ \\
\hline $\mathrm{FEV}_{1} / \mathrm{FVC}$ ratio & $38.45(11.08)$ & $(31.01,45.90)$ & $31.80(7.42)$ & $(26.49,37.11)$ \\
\hline Total lung capacity, \% predicted & $105.20(16.38)$ & $(92.63,117.80)$ & $114.10(17.02)$ & $(101.90,126.30)$ \\
\hline Residual volume, \% predicted & $178.70(45.02)$ & $(144.10,213.30)$ & $180.90(34.95)$ & $(155.90,205.90)$ \\
\hline Diffusing capacity, \% predicted & $42.27(12.92)$ & $(33.60,50.95)$ & $46.90(10.09)$ & $(39.68,54.12)$ \\
\hline \multicolumn{5}{|l|}{ Incremental exercise } \\
\hline $\mathrm{VO}_{2}, \%$ predicted & $37.91(14.16)$ & $(28.40,47.42)$ & $39.50(11.10)$ & $(31.56,47.44)$ \\
\hline Peak workload (Watts) & $49.09(22.56)$ & $(33.93,64.25)$ & $48.00(16.86)$ & $(35.94,60.06)$ \\
\hline Peak heart rate, $\%$ predicted & $76.36(9.7 I)$ & $(69.84,82.88)$ & $79.60(8.72)$ & $(73.36,85.84)$ \\
\hline Peak ventilation, \% predicted & $99.36(23.32)$ & $(83.70,115.00)$ & $93.70(13.12)$ & $(84.32,103.10)$ \\
\hline Baseline dyspnea index & $5.91(1.76)$ & $(4.73,7.09)$ & $5.30(1.16)$ & $(4.47,6.13)$ \\
\hline
\end{tabular}

Note: Values are means followed by (standard deviation).

Abbreviations: $\mathrm{Cl}$, confidence intervals; $\mathrm{FEV}_{1}$, forced expiratory volume in I second; $\mathrm{FVC}$, forced vital capacity; $\mathrm{VO}_{2}$, oxygen consumption.

on the ten patients who completed the theophylline arm of the study to assess whether any factors on pre-treatment pulmonary function or exercise data that could identify patients who might benefit from theophylline, via regression analysis (Table 3). We found that neither baseline $\mathrm{FEV}_{1}$, FVC, residual volume, dyspnea severity (as assessed by baseline dyspnea index), nor any resting exercise parameters predicted the

Table 3 Correlations between the baseline pulmonary function and exercise measurements with change in exercise duration (\% change)

\begin{tabular}{|c|c|}
\hline & $\begin{array}{l}\text { Pearson correlation coefficient } \\
(P \text {-value })\end{array}$ \\
\hline $\mathrm{FEV}_{1}, \%$ predicted & $0.38(0.283)$ \\
\hline FVC, \% predicted & $0.297(0.404)$ \\
\hline TLC, \% predicted & $0.35(0.327)$ \\
\hline RV, \% predicted & $0.219(0.544)$ \\
\hline DLCO, \% predicted & $0.87(0.001)$ \\
\hline Peak $\mathrm{VO}_{2}, \%$ predicted & $0.37(0.290)$ \\
\hline Peak $\mathrm{VO}_{2} / \mathrm{kg}, \%$ predicted & $0.297(0.404)$ \\
\hline Peak HR, \% predicted & $0.364(0.301)$ \\
\hline Peak VE, \% predicted & $0.135(0.710)$ \\
\hline \multicolumn{2}{|c|}{$\begin{array}{l}\text { Notes: The Pearson correlation coefficient was used to assess the strength of } \\
\text { linear association between each variable and the percent change in exercise duration } \\
\text { on theophylline. Diffusing capacity showed a strong positive correlation with the } \\
\text { percent change in exercise duration ( } \mathrm{r}=0.87 \text { ). For other variables, there was no } \\
\text { evidence of having a significant linear association with change in exercise duration. } \\
\text { Abbreviations: } \mathrm{Cl} \text {, confidence intervals; } \mathrm{DLCO} \text {, diffusing capacity; FEV }{ }_{1} \text {, forced } \\
\text { expiratory volume in I second; FVC, forced vital capacity; HR, heart rate; IC, } \\
\text { inhibitory capacity; RV, residual volume; TLC, total lung capacity; VE, ventilatory } \\
\text { efficiency; } \mathrm{VO}_{2} \text {, oxygen consumption. }\end{array}$} \\
\hline
\end{tabular}

magnitude of response to theophylline. However, we did find a positive correlation between diffusing capacity and improvements in exercise duration on theophylline $(r=0.87)$, meaning that subjects with greater diffusing capacity tended to demonstrate greater improvements in exercise capacity on theophylline. This is a novel finding and could potentially help predict which patients are more likely to respond to theophylline. However, we acknowledge that one positive correlation among nine separate univariate analyses could occur by chance and confirmation with future studies is required before any definitive conclusions could be drawn.

The reduction in peak ventilation is of uncertain significance. In the context of a trend toward improvement in exercise capacity, it is possible that the reduction in ventilation represents improved gas exchange and ventilatory efficiency (lower ventilatory efficiency/oxygen consumption ratio). However, it should be noted that this finding was not observed in previous exercise studies with theophylline $e^{6,10}$ and consequently, confirmation with a larger study is required.

A post-hoc analysis using our study's data suggests that a total of 90 patients (not accounting for dropouts) would have been required to have $80 \%$ power to detect a difference in exercise duration of $26 \%$. Our study's relatively small sample size did not allow us to detect potential effects of theophylline on pulmonary function or other exercise parameters, such as heart rate response. 
Since the conception of our study, some authors have suggested the combination of theophylline and inhaled corticosteroids may have a modest improvement on some measures of inflammation and lung function. ${ }^{18}$ It is notable that all but one of our study subjects was on inhaled steroids concurrently, although our protocol did not require use of inhaled corticosteroids for study inclusion. The possibility that there may be a beneficial interaction between theophylline and inhaled steroids is interesting but ultimately does not change the conclusions of our study, as we were not able to detect a significant improvement in exercise capacity with the addition of theophylline. Reanalysis of our study results excluding the one patient not taking inhaled corticosteroids did not significantly change our study results.

\section{Conclusion}

Our study did not find a statistically significant improvement in exercise duration with theophylline compared to placebo, in patients with COPD already treated with combination long-acting inhaled bronchodilator therapy. Although there was a mean improvement in exercise duration of $26 \%$, the result was not statistically significant due to large variations in individual patient responses to theophylline. Our results do however suggest that further investigation is warranted on the issue. In our study, theophylline was tolerated by the majority of subjects, and four of 10 patients able to tolerate theophylline did demonstrate a clinically meaningful improvement in exercise capacity. Our small sample size precludes any definitive conclusions on the utility of theophylline but if our results are validated in a larger study, this would have important clinical implications on the treatment of COPD, particularly given the low cost and accessibility of theophylline.

This is the first study to evaluate the effect of theophylline on exercise performance in COPD patients already on long-acting anticholinergic and beta-agonist therapy. Our results suggest that some patients may experience a clinically significant improvement in exercise capacity with theophylline, but further study is needed before definitive conclusions may be drawn.

\section{Acknowledgments}

This study was funded by an Ontario Thoracic Society block term grant.

\section{Author contributions}

NV designed the study, participated in subject assessment and conduct of exercise testing, and is the primary author of the manuscript. GA supervised theophylline dosing and participated in the writing of the manuscript. KA participated in patient selection and screening, supervised theophylline dosing, and reviewed the manuscript. CT conducted pulmonary function testing and exercise testing and was responsible for data management. ES performed the statistical analysis and participated in the writing of the manuscript. SA contributed to the design of the study and participated in the writing of the manuscript. The authors would like to thank Crystal Lameira and Lisa Charrette for their assistance in the preparation of this manuscript.

\section{Disclosures}

NV has received speaking fees from AstraZeneca and GlaxoSmithKline and an unrestricted educational grant from Boehringer Ingelheim. The other authors report no conflicts of interest in this work.

\section{References}

1. Rennard SI, Anderson W, ZuWallck R, et al. Use of a long-acting inhaled $\beta_{2}$-adrenergic agonist, salmeterol xinafoate, in patients with chronic obstructive pulmonary disease. Am J Respir Crit Care Med. 2001;163:1087-1092.

2. Casaburi R, Mahler DA, Jones P, et al. A long-term evaluation of oncedaily inhaled tiotropium in chronic obstructive pulmonary disease. Eur Respir J. 2002;19:217-224.

3. Vincken W, van Noord JA, Greefhorst APM, Bantje ThA, Kesten S, Korducki L. Improved health outcomes in patients with COPD during 1 yr's treatment with tiotropium. Eur Respir J. 2002;19:209-216.

4. Rabe KF, Hurd S, Anzueto A, et al. Global strategy for the diagnosis, management, and prevention of chronic obstructive pulmonary disease: GOLD Executive Summary. Am J Respir Crit Care Med. 2007;176: 532-555.

5. O'Donnell DE, Aaron S, Bourbeau J, et al. Canadian Thoracic Society recommendations for management of chronic obstructive pulmonary disease - 2007 update. Can Respir J. 2007;14(Suppl B):5B-32B.

6. Fink G, Kaye C, Sulkes J, et al. Effects of theophylline on exercise performance in patients with severe chronic obstructive pulmonary disease. Thorax. 1994;49:332-334.

7. Shannon M. Predictors of major toxicity after theophylline overdose. Ann Intern Med. 1993;119:1161-1167.

8. Zuwallack RL, Mahler DA, Reilly D, Church N, Emmett A, Richard K. Salmeterol plus theophylline combination therapy in the treatment of COPD. Chest. 2001;119(6):1628-1630.

9. Cazzola M, Matera MG. The additive effect of theophylline on a combination of formoterol and tiotropium in stable COPD: A pilot study. Respir Med. 2007;101:957-962.

10. McKay SE, Howie CA, Thomson AH, Whiting B, Addis GJ. Value of theophylline treatment in patients handicapped by chronic obstructive lung disease. Thorax. 1993;48:227-232.

11. Tsukino M, Nishimura K, Ikeda A, Hajiro T, Koyama H, Izumi T. Effects of theophylline and ipratropium bromide on exercise performance in patients with stable chronic obstructive pulmonary disease. Thorax. 1998;53:269-273

12. Liesker J, Wijkstra P, Ten Hacken N, Koeter G, Postma D, Kerstjens H. A systemic review of the effects of bronchodilators on exercise capacity in patients with COPD. Chest. 2002;121:597-608.

13. American Thoracic Society; American College of Chest Physicians. ATS/ACCP Statement on cardio-pulmonary exercise testing. Am J Respir Crit Care Med. 2003;167 (2):211-277. Erratum in: Am J Respir Crit Care Med. 2003:1451-1452. 
14. O’Donnell DE, Voduc N, Fitzpatrick M, Webb K. Effect of salmeterol on the ventilatory response to exercise in chronic obstructive pulmonary disease. Eur Respir J. 2004;24:86-94.

15. O'Donnell DE, Fluge T, Gerken F, et al. Effects of tiotropium on lung hypertension, dyspnea, and exercise tolerance. Eur Respir J. 2004;23:832-840.

16. Berton DC, Reis M, Sigueira AC, et al. Effects of tiotropium on dynamic hypertension and exercise endurance in COPD. Respir Med. 2010;104:1288-1296.
17. Puente-Maestu L, Villar F, de Miguel J, et al. Clinical relevance of constant power exercise duration changes in COPD. Eur Respir J. 2009;34(2):340-345.

18. Ford PA, Durham AL, Russell RE, Gordon F, Adcock IM, Barnes PJ. Treatment of low-dose theophylline combined with an inhaled corticosteroid in COPD. Chest. 2010;137(6):1338-1344.

\section{Publish your work in this journal}

The International Journal of COPD is an international, peer-reviewed journal of therapeutics and pharmacology focusing on concise rapid reporting of clinical studies and reviews in COPD. Special focus is given to the pathophysiological processes underlying the disease, intervention programs, patient focused education, and self management protocols.

\section{Dovepress}

This journal is indexed on PubMed Central, MedLine and CAS. The manuscript management system is completely online and includes a very quick and fair peer-review system, which is all easy to use. Visit $\mathrm{http}: / / \mathrm{www}$.dovepress.com/testimonials.php to read real quotes from published authors.

Submit your manuscript here: http://www.dovepress.com/international-journal-of-copd-journal 